\title{
Estimating Friction Parameters in Reaction Wheels for Attitude Control
}

\author{
Valdemir Carrara and Hélio Koiti Kuga \\ INPE, Avenida dos Astronautas 1758, 12227-010 São José dos Campos, SP, Brazil \\ Correspondence should be addressed to Valdemir Carrara; val.carrara@gmail.com
}

Received 11 March 2013; Accepted 12 May 2013

Academic Editor: Antonio F. Bertachini A. Prado

Copyright (C) 2013 V. Carrara and H. K. Kuga. This is an open access article distributed under the Creative Commons Attribution License, which permits unrestricted use, distribution, and reproduction in any medium, provided the original work is properly cited.

\begin{abstract}
The ever-increasing use of artificial satellites in both the study of terrestrial and space phenomena demands a search for increasingly accurate and reliable pointing systems. It is common nowadays to employ reaction wheels for attitude control that provide wide range of torque magnitude, high reliability, and little power consumption. However, the bearing friction causes the response of wheel to be nonlinear, which may compromise the stability and precision of the control system as a whole. This work presents a characterization of a typical reaction wheel of $0.65 \mathrm{Nms}$ maximum angular momentum storage, in order to estimate their friction parameters. It used a friction model that takes into account the Coulomb friction, viscous friction, and static friction, according to the Stribeck formulation. The parameters were estimated by means of a nonlinear batch least squares procedure, from data raised experimentally. The results have shown wide agreement with the experimental data and were also close to a deterministic model, previously obtained for this wheel. This model was then employed in a Dynamic Model Compensator (DMC) control, which successfully reduced the attitude steady state error of an instrumented one-axis air-bearing table.
\end{abstract}

\section{Introduction}

This paper presents a Dynamic Model Compensator (DMC) control of a reaction wheel in current control mode. The error is compensated by means of a mathematical model of the wheel dynamics and the bearing friction. Reaction wheels are actuators largely employed in attitude control subsystems in order to provide attitude pointing and stability of artificial satellites. They consist of a Brushless DC (BLDC) motor coupled to a high inertia flywheel. The torque applied to the wheel is sensed by the satellite in the opposite direction, allowing the attitude control based on information of inertial sensors like gyroscopes, sun sensors, magnetometers, and star sensors. Reaction wheels are devices that must operate continuously for several years in vacuum conditions, subjected to wide variations in temperature and high radiation doses. So, its reliability and quality are essential to the satellite health. These requirements pose great challenge to a reaction wheel design, which makes such equipment highly complex and expensive. Reaction wheels are classified according to their capability of storing angular momentum, from the small ones employed in microsatellites to large ones appropriated for orbital stations and communication satellites. Normally reaction wheels are operated either in current (or equivalently torque) mode or in speed mode. In current mode the electronics delivers the necessary current to the motor in order to achieve the commanded torque. In speed mode a secondary outer control loop regulates the current to eliminate the error between the commanded angular speed and the flywheel speed, which is measured by some sort of rate sensor (usually Hall effect sensor or optical incremental encoder). The speed mode control avoids the bearing friction effects, which causes a nonlinear behavior in the current control mode. However, speed control introduces more complexity in the electronics and also causes some delay in the wheel response. In order to assure linearity in the current mode and eventually disregard the speed control mode, this work suggests mitigating the effects of friction by adopting a DMC controller in current control loop. This compensator was applied to an off-the-shelf reaction wheel that operates in both current, and speed mode. The friction model includes Coulomb, viscous, and static, or breakaway torques. With the aim of evaluating the control performance, the static 
friction was replaced by the Stribeck friction, which, unlike the previous one, does not present discontinuities when the motor reverses its rotation sense. All friction parameters and the motor coefficient were obtained by a least squares fit of data collected from several experiments performed with the wheel in current mode. The experiments consisted of a continuously varying current command in order to stimulate the wheel through various speeds and sense inversions, so as to assure correct parameters identification and model fidelity. The DMC was then introduced in the attitude control loop of an air-bearing table that emulates the frictionless conditions found in space. The table has a fiber optic gyroscope for measuring angular rate (that provides the reference for the attitude after integration), the reaction wheel, a system of radio-modem for reaction wheel telemetry and command, and a power supply battery. A small computer fan was placed in the air-bearing table, so as to yield a small torque, which will be dully balanced by the attitude control procedure. By proper selection of the initial conditions plus the fan torque, the wheel will be forced by the attitude control to reverse its direction of rotation. The results show that there is a significant gain when the DMC is implemented in the control loop, when compared with the simple current mode control, with control performance comparable to the speed mode. A comparison of the statistical method for determining friction and motor parameters with a deterministic method, in which each parameter has been obtained from a dedicated experiment to highlight its influence, is also presented in this study.

In an interesting work, Robertson and Stoneking [1] state that the Guidance, navigation and control subsystem (or sometimes attitude and orbit control subsystem) presents a high number of critical failures in satellites when compared with other subsystems. From these failures, almost 30\% can be assigned to the reaction wheel, which confirms that a good wheel design or selection makes difference. In Carrara and Milani [2] the friction parameters of a reaction wheel commanded in current are raised from experimental means. In that work the wheel is subject to specific commands in order to highlight a particular parameter. These are then calculated by manual curve adjustment, based on minimum quadratic variation. The model used took into account the Coulomb and viscous frictions. In Carrara [3] the same model was used in an attitude controller of an air-bearing table. The controller used command in current with dynamic compensation based only on Coulomb and viscous frictions. With this method it was possible to reduce the error during wheel rotation inversion by an order of magnitude. Later, a comparison between the two forms of control in Carrara et al. was made [4], which showed that the dynamic compensator introduces an error comparable but slightly higher to control mode in the angular velocity of the wheel.

The wheel friction parameters plus the Stribeck friction (in fact, a continuous and differentiable model of static friction or departure friction) were estimated by a Kalman filter in Fernandes et al. [5] but with nonconclusive results, because of the scarcity of accurate experimental data. Few works in the literature relate friction models with reaction wheels bearings $[6,7]$. On the other hand, several papers have

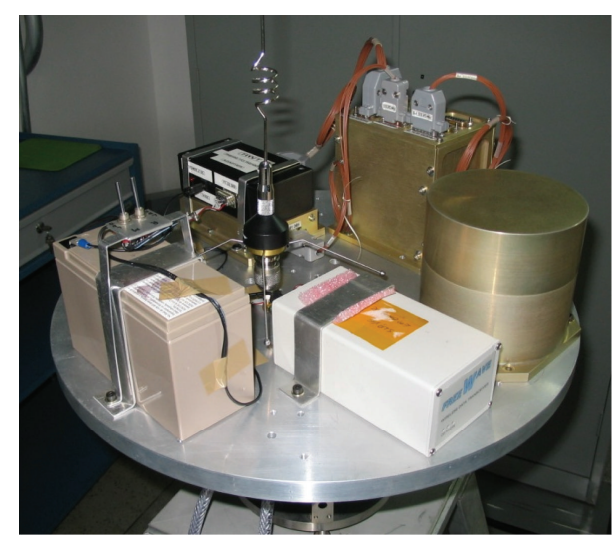

FIGURE 1: Experiment mounted on the air-bearing table.

friction models and estimation of parameters in rotors, as in Olsson et al. [8] and Canudas de Wit and Ge [9], including a dynamic model for friction by Canudas de Wit et al. [10], and Canudas de Wit and Lischinsky [11], both based on the LuGre (Lund-Grenoble) friction model [12]. This dynamic model was later employed in Carrara et al. [13] to estimate the parameters of a reaction wheel by Kalman filtering. A model of the slip-stick phenomenon was simulated by Karnopp [14], while Al-Bender et al. [15] presented a dynamic model based on the generalized Maxwell-slip friction. Hirschorn and Miller [16] proposed a dynamic controller for system with a bristle model for nonlinear friction effects. Amin et al. [17] suggest using a nonlinear observer to estimate and to compensate for the friction, which presented good concordance with experimental data. Hensen et al. [18] experimentally obtained the friction parameters of the dynamic LuGre model by means of an apparatus, and Ramasubramanian and Ray [19] employed the extended Kalman-Bucy filter (EKBF) to estimate the Dahl model friction coefficients. ArmstrongHélouvry et al. [20] compiled the existing friction models and compensation methods in a comprehensive work.

This work proposes to estimate, by means of a nonlinear least squares procedure, the parameters of the friction of a reaction wheel, shown in the photo of Figure 1, considering not only the Coulomb and viscous frictions but also the Stribeck friction. The so-estimated parameters are then compared to those obtained in Carrara and Milani [2] and Carrara [3]. In the following sections, the formulation of friction model and of the estimation of parameters will be presented. The experimental results appear next, together with the comparison between both methods: statistical and deterministic. The conclusions are presented in sequence. A typical reaction wheel [21] acquired by the Space Mechanics and Control Division of National Institute for Space Research (INPE) was employed for data acquisition and analysis.

\section{Mathematical Model}

For gathering the necessary data for this work, a setup made by Carrara and Milani [2] was used. In a bearing table system of one degree of freedom in rotation (Figure 1) a reaction wheel with maximum capacity of $0.65 \mathrm{Nms}$ commanded by 


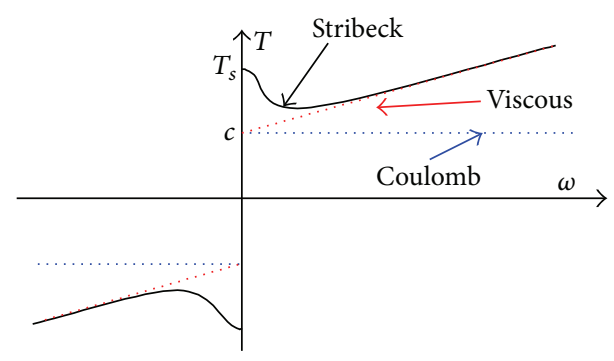

FIGURE 2: Friction torque model used in the parameter estimation.

current via serial interface, a fiber optic gyroscope of one axis (not used in this work), a command and telemetry electronics, a radio modem for communication with the equipment, and a battery for power supply were installed. The programs needed to command the wheel and make the current readings and angular velocity were written in $\mathrm{C}++$ and run on a computer that is external to the table.

The mathematical model of a reaction wheel is analogous to the model of a DC motor, in which inertia includes, besides the rotor inertia, the inertia of the flywheel attached to the axis of the wheel. In the model considered here, the viscous friction, Coulomb friction, and the friction of Stribeck were included. The differential equation describing the motion is

$$
T_{w}=J_{w} \dot{\omega}+b \omega+\operatorname{sgn}(\omega)\left[c+d e^{-\omega^{2} / \omega_{s}^{2}}\right],
$$

where $T_{w}$ is the motor torque, the wheel's and rotor inertia is $J_{w}, b$ is the coefficient of viscous friction, $c$ is the Coulomb friction torque, $d$ is the starting torque, $\omega$ is the angular velocity of the wheel, and $\omega_{s}$ is known as Stribeck speed $[8,9]$. The torque model is displayed graphically in Figure 2. The starting torque $d$ can be decomposed on the difference between the static torque $T_{s}$ and the Coulomb torque $c$; that is, $d=T_{s}-c$. Neglecting nonlinear effects present in current to torque conversion, one can consider that the torque applied to the motor is proportional to the current in the stator, $I$, in the form

$$
T_{w}=k_{m} I
$$

In current control mode, one commands the current $I$ on the wheel and gets telemetry readings of angular velocity $\omega$ and current itself, which may be slightly different from that commanded due to the presence of an internal current control loop to the wheel. For the estimation of parameters by means of a least squares procedure, the state to solve for is composed of the angular velocity, the motor constant, viscous friction coefficient, Coulomb torque and static torque. Since the inertia of the wheel cannot be estimated independently of other parameters, the inertia value supplied by the manufacturer of $J_{w}=1.5 \times 10^{-3} \mathrm{~kg} \mathrm{~m}^{2}$ was adopted. The state to be estimated is then

$$
\begin{aligned}
\mathbf{x} & =\left(\begin{array}{lllll}
\omega & \frac{k_{m}}{J_{w}} & \frac{b}{J_{w}} & \frac{c}{J_{w}} & \frac{T_{s}}{J_{w}}
\end{array}\right)^{T} \\
& =\left(\begin{array}{lllll}
x_{1} & x_{2} & x_{3} & x_{4} & x_{5}
\end{array}\right)^{T} .
\end{aligned}
$$

Stribeck speed $\omega_{s}$ could also be estimated, but preliminary tests showed that the noise present in the measurements at low speed, where this parameter is important, does not allow a good estimate of its value. In addition the estimated values of the remaining parameters are barely affected by $\omega_{s}$. As a result one adopted to this speed the $4 \mathrm{rpm}$ value obtained indirectly through a mapping of the average current as a function of the angular velocity of the wheel at low speeds, using the speed control mode.

From (1), the dynamical model for the estimation process is drawn:

$$
\dot{x}_{1}=x_{2} I-x_{3} x_{1}-\operatorname{sgn}\left(x_{1}\right)\left[x_{4}+\left(x_{5}-x_{4}\right) e^{-x_{1}^{2} / \omega_{s}^{2}}\right] .
$$

Once the dynamical part is represented by only one (time) variable (rotation $x_{1}$ ), and the remaining states are parameters, the nonnull elements of the corresponding Jacobian matrix of partial derivatives are

$$
\begin{gathered}
\frac{\partial \dot{x}_{1}}{\partial x_{1}}=-x_{3}+2 \frac{x_{1}}{\omega_{s}^{2}} \operatorname{sgn}\left(x_{1}\right)\left(x_{5}-x_{4}\right) e^{-x_{1}^{2} / \omega_{s}^{2}}, \\
\frac{\partial \dot{x}_{1}}{\partial x_{2}}=I \\
\frac{\partial \dot{x}_{1}}{\partial x_{3}}=-x_{1} \\
\frac{\partial \dot{x}_{1}}{\partial x_{4}}=-\operatorname{sgn}\left(x_{1}\right)\left(1-e^{-x_{1}^{2} / \omega_{s}^{2}}\right) \\
\frac{\partial \dot{x}_{1}}{\partial x_{5}}=-\operatorname{sgn}\left(x_{1}\right) e^{-x_{1}^{2} / \omega_{s}^{2}} .
\end{gathered}
$$

The data were generated with the wheel subjected to two command profiles, both with small amplitude, so as to keep it at low speeds and with periodic reversals in the direction of rotation, as shown in Figure 3. The first profile consisted of multiple sinusoidal cycles in which each period had the amplitude and the period chosen randomly within certain limits. The second profile had random amplitude, constant current in each actuation, and reverse direction every 30 seconds, similar to a square wave.

The bearing temperature and atmospheric pressure inside the reaction wheel were monitored during the entire run of the profiles, with duration of 300 seconds each. Although it is plausible that the temperature affects the friction and, as a consequence, the behavior of the wheel, this influence has not been taken into account in this model, since the variation of both during the experiment was small, less than $1^{\circ} \mathrm{C}$ in temperature. Note that, particularly in Figure 3(b), the Coulomb torque causes changes of inflection in the curve of the angular velocity when it reverses its direction of rotation. This is an indication that these experiments are able to provide information for this and other estimation parameters, which will be presented in the following section.

\section{Estimation Procedure}

The procedure of parameter estimation from (1) was based on the batch least squares method. The weighted loss function $J$, 


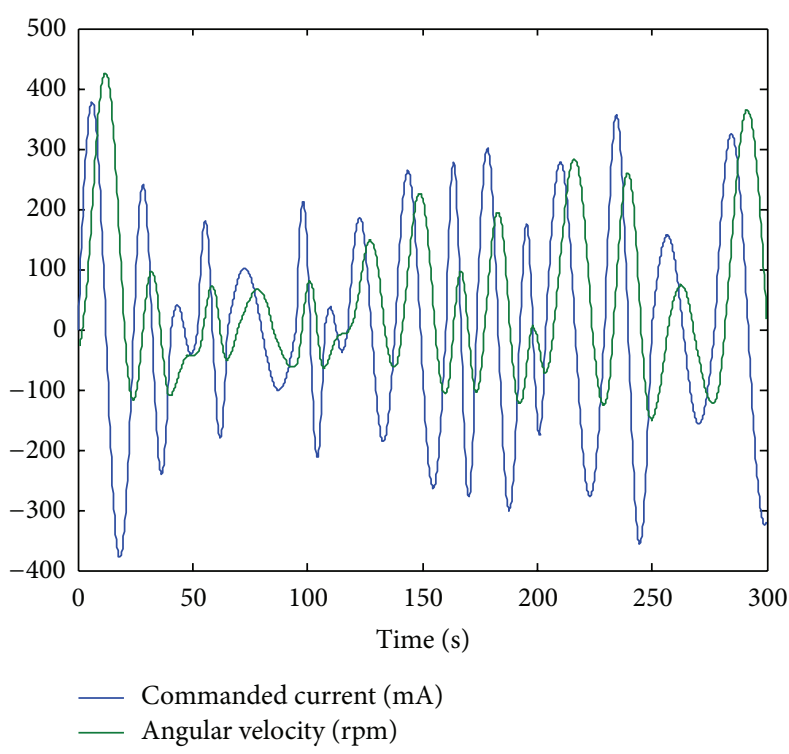

(a)

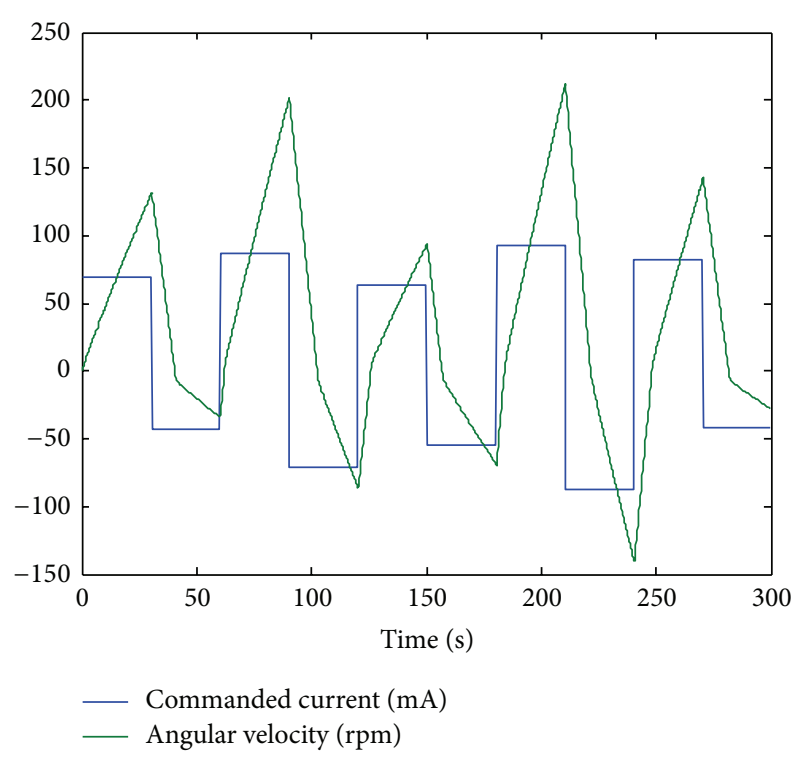

(b)

Figure 3: Sinusoidal (a) and stepwise (b) profiles commanded to the reaction wheel.

considering a priori information, in norm notation, is given by

$$
J=\|\mathbf{y}-\mathbf{H} \mathbf{x}\|_{\mathbf{R}^{-1}}^{2}+\left\|\widehat{\mathbf{x}}_{o}-\mathbf{x}\right\|_{\mathbf{P}_{o}^{-1}}^{2},
$$

where $\|\cdot\|$ represents the norm of a matrix or vector, $\mathbf{y}$ is the vector containing $m$ measurements, $\mathbf{H}$ is the $m \times n$ matrix that relates the measurements to the state $\mathbf{x}$ of $n$ elements, $\widehat{\mathbf{x}}_{o}$ is the a priori state value, $\mathbf{R}$ is the $m \times m$ covariance matrix of measurement errors, and $\mathbf{P}_{o}$ is the covariance matrix of the errors on the a priori state. Initially the loss function is in the form

$$
J=\left\|\left[\begin{array}{c}
\left(\mathbf{P}_{o}^{-1}\right)^{1 / 2} \widehat{\mathbf{x}}_{o} \\
\left(\mathbf{R}^{-1}\right)^{1 / 2} \mathbf{y}
\end{array}\right]-\left[\begin{array}{c}
\left(\mathbf{P}_{o}^{-1}\right)^{1 / 2} \\
\left(\mathbf{R}^{-1}\right)^{1 / 2} \mathbf{H}
\end{array}\right] \mathbf{x}\right\|^{2}
$$

where $(\cdot)^{1 / 2}$ represents a square root matrix of $(\cdot)$. Utilizing an orthogonal transformation $\mathbf{T}$ of, for example, Householder, that does not change the norm, one triangularizes the system so that:

$$
\begin{aligned}
J & =\left\|\mathbf{T}\left[\begin{array}{c}
\left(\mathbf{P}_{o}^{-1}\right)^{1 / 2} \widehat{\mathbf{x}}_{o} \\
\left(\mathbf{R}^{-1}\right)^{1 / 2} \mathbf{y}
\end{array}\right]-\mathbf{T}\left[\begin{array}{c}
\left(\mathbf{P}_{o}^{-1}\right)^{1 / 2} \\
\left(\mathbf{R}^{-1}\right)^{1 / 2} \mathbf{H}
\end{array}\right] \mathbf{x}\right\|^{2} \\
& =\left\|\left[\begin{array}{c}
\mathbf{y}_{1} \\
\mathbf{y}_{2}
\end{array}\right]-\left[\begin{array}{c}
\mathbf{H}_{1} \\
\mathbf{0}
\end{array}\right] \mathbf{x}\right\|^{2}=\left\|\mathbf{y}_{1}-\mathbf{H}_{1} \mathbf{x}\right\|^{2}+\left\|\mathbf{y}_{2}\right\|^{2} .
\end{aligned}
$$

Note that after the orthogonal transformation, $\mathbf{H}_{1}$ is a $n \times n$ triangular matrix, $\mathbf{0}$ is a $m \times n$ matrix of zeros, and $\mathbf{y}_{1}$ and $\mathbf{y}_{2}$ are vectors of sizes $n$ and $m$, respectively, resulting from the application of the orthogonal transformation $\mathbf{T}$. Therefore the minimum of the loss function is simply

$$
J_{\min }=\left\|\mathbf{y}_{2}\right\|^{2} \text { if }\left\|\mathbf{y}_{1}-\mathbf{H}_{1} \mathbf{x}\right\|=0,
$$

which is the least squares solution according to Lawson and Hanson [22]. Once the matrix $\mathbf{H}_{1}$ is triangular, the resolution of

$$
\mathbf{y}_{1}=\mathbf{H}_{1} \widehat{\mathbf{x}}
$$

is trivial (back substitution), and $\widehat{\mathbf{x}}$ is the estimated state vector. This approach was coded in Fortran and adapted [23] to solve the nonlinear problem of estimation of friction parameters. In this case, $\mathbf{H}$ is the matrix of partial derivatives with components given by (5), and $\mathbf{y}, \mathbf{x}$, and $\widehat{\mathbf{x}}_{o}$ are now deviations from the nominal. Because of the nonlinear nature of the problem, from an initial condition $\widehat{\mathbf{x}}_{o}$ (a priori), the solution is obtained iteratively and converges quickly in few iterations.

\section{Estimation of Friction Parameters}

Some of the friction parameters of this wheel were estimated by Carrara and Milani [2] and Carrara [3] in previous works. Because very specific methods for individual computation of the friction parameters were used in those works, they were named deterministic methods, in contrast with the statistical method employed in this study. By the deterministic methods, the viscous friction coefficient $b=5.16 \times 10^{-6} \mathrm{Nms}$, the Coulomb friction $c=0.8795 \times 10^{-3} \mathrm{Nm}$, and the motor constant $k_{m}=0.0270 \mathrm{Nm} / \mathrm{A}$ were obtained.

In the parameter estimation procedure the departure state vector was set to

$$
\mathbf{x}_{o}=\left(\begin{array}{lllll}
0 & 18 & 0.00344 & 0.5863 & 0
\end{array}\right)^{T}
$$

which corresponds to the values of the deterministic methods, as defined by (3). The profile 2 was used to estimate the values of the parameters $x_{2}, x_{3}, x_{4}$, and $x_{5}$. The profile 
TABLE 1: Friction parameters of the reaction wheel.

\begin{tabular}{lccc}
\hline \multicolumn{1}{c}{ Parameter } & & Deterministic & Statistical \\
\hline Motor constant & $k_{m}$ & 0.0270 & 0.0228 \\
Viscous coefficient & $b$ & $5.16 \times 10^{-6}$ & $4.83 \times 10^{-6}$ \\
Coulomb torque & $c$ & $0.8795 \times 10^{-3}$ & $0.8795 \times 10^{-3}$ \\
Static torque & $T_{s}$ & - & $0.9055 \times 10^{-3}$ \\
\hline
\end{tabular}

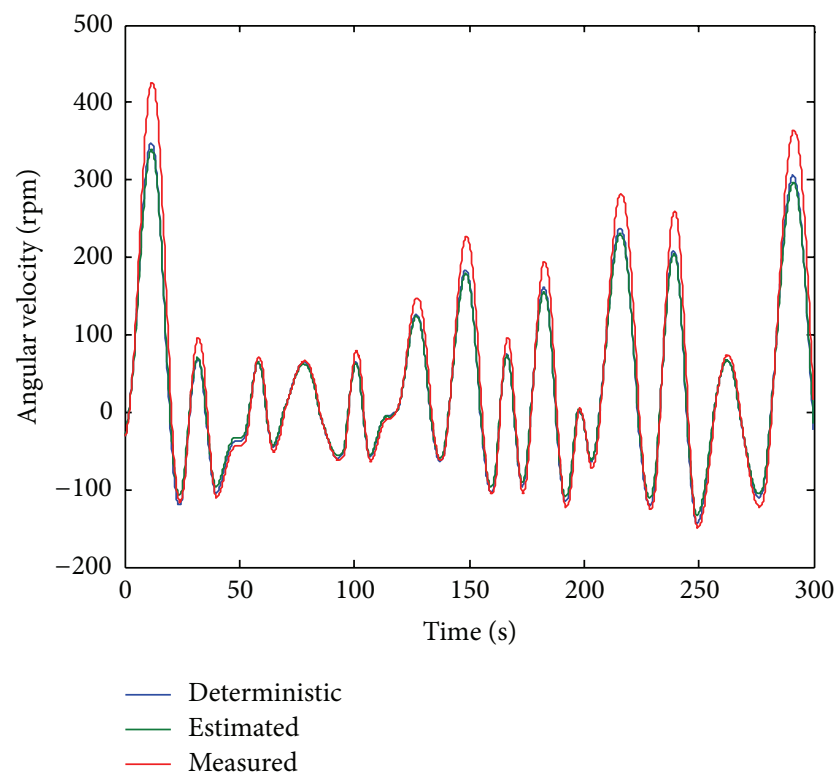

FIGURE 4: Results of deterministic and statistical methods in the profile 1.

1 (sinusoidal) was used to validate the estimated parameters. In the least squares procedure one assumed that rotation measurements had a standard deviation of about $5 \mathrm{rpm}$. The state vector after convergence of the procedure was

$$
\widehat{\mathbf{x}}_{o}=\left(\begin{array}{lllll}
0 & 15.205 & 0.00322 & 0.5863 & 0.6037
\end{array}\right)^{T} .
$$

Considering the inertia value $J_{w}=1.5 \times 10^{-3} \mathrm{~kg} \mathrm{~m}^{2}$, the friction parameters result in $b=4.83 \times 10^{-6} \mathrm{Nms}, c=0.8795 \times$ $10^{-3} \mathrm{Nm}$, and $k_{m}=0.0228 \mathrm{Nm} / \mathrm{A}$. Table 1 shows the results obtained herein. It is realized that the highest difference was encountered in the motor constant, which was $15 \%$ below the deterministic method. The Coulomb torque did not present meaningful difference within the accuracy tolerance adopted in its computation.

Figure 4 shows to profile 1 a comparison of the measured (Figure 3) and estimated speeds by the deterministic and statistical methods. Note that both methods present similar results; however, the error with respect to actual measurements is still relatively high.

Figure 5 shows the same results for the stepwise profile 2 (Figure 4). Note clearly the better closeness of the statistical adjustment (compared to the deterministic one) to the experimental rotation measurements.

Figure 6 shows the measurements (in red) and the residuals between the measured rotations and the values

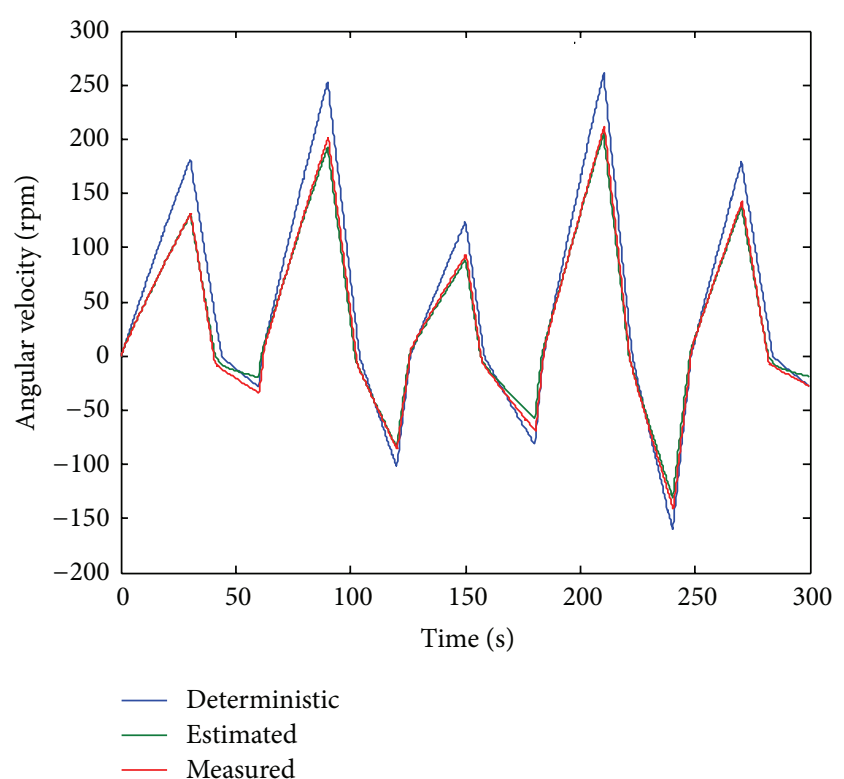

FIgURE 5: Results of deterministic and statistical methods in the profile 2.

estimated by both methods (deterministic and statistical), for the sinusoidal profile 1 . Increasing residuals near the zero crossings is verified, where the friction models have lower performance.

Figure 7 shows the residuals between measured and estimated rotations for the stepwise profile 2 . The best performance of the statistical adjustment at low speeds (50$100 \mathrm{rpm}$ ) is also quite pronounced.

The results indicate problems in the wheel response at low angular rates, mainly in transitions crossing the zero level. Nevertheless, the model obtained by the statistical estimation of parameters behaves better in this range. In practical terms, this model, when used in a control system, provides a smooth transition through zero and can eliminate the need to define a dead zone, facilitating the design and implementation of the control system. On the other hand it should be noted that the mathematical model used in both methods is symmetrical with respect to the direction of rotation. However there is evidence [24] that bearings may be asymmetric, although the degree of asymmetry is in general small.

4.1. Torque Mode Control. In order to emphasize the nonlinear friction effect in the controller performance, a cooler fan was attached to the air-bearing table and oriented in such a way that it introduces a small but constant torque. The initial velocity of the wheel was adjusted so that a zero-speed crossover occurs during the control action. A PID controller was used to control the attitude of the air-bearing table, based on the integrated signal of the FOG gyro. The PID gains were adjusted to minimize or to avoid the overshoot response in attitude and were kept constant during the whole experiment. The air-bearing table dynamics can be modeled as a one-axis rigid body with inertia $J$ and the fan disturbance torque $T_{d}$ :

$$
J \dot{\Omega}=T_{w}+T_{d},
$$




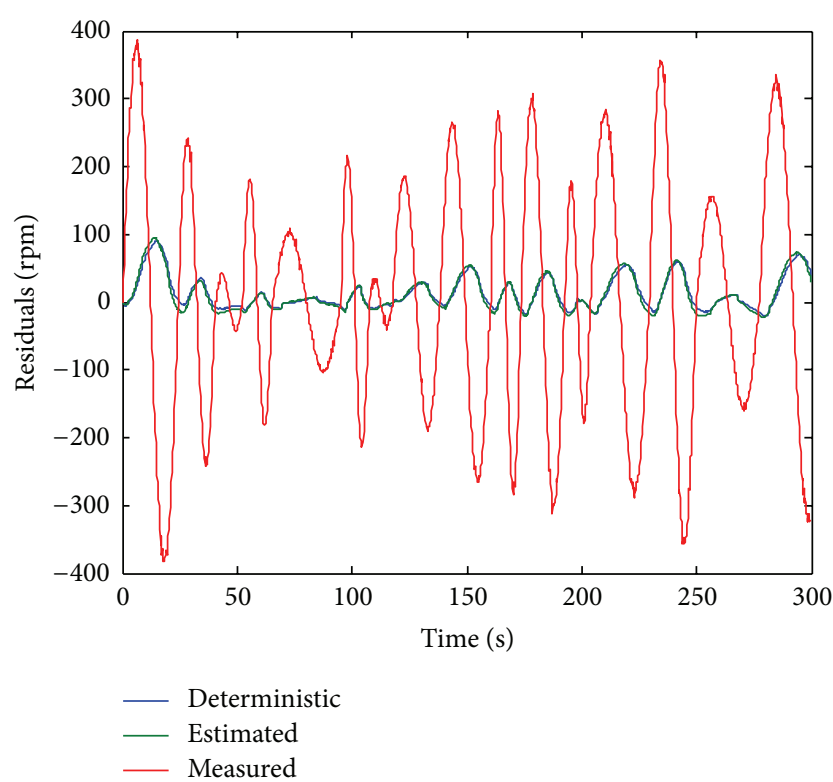

(a)

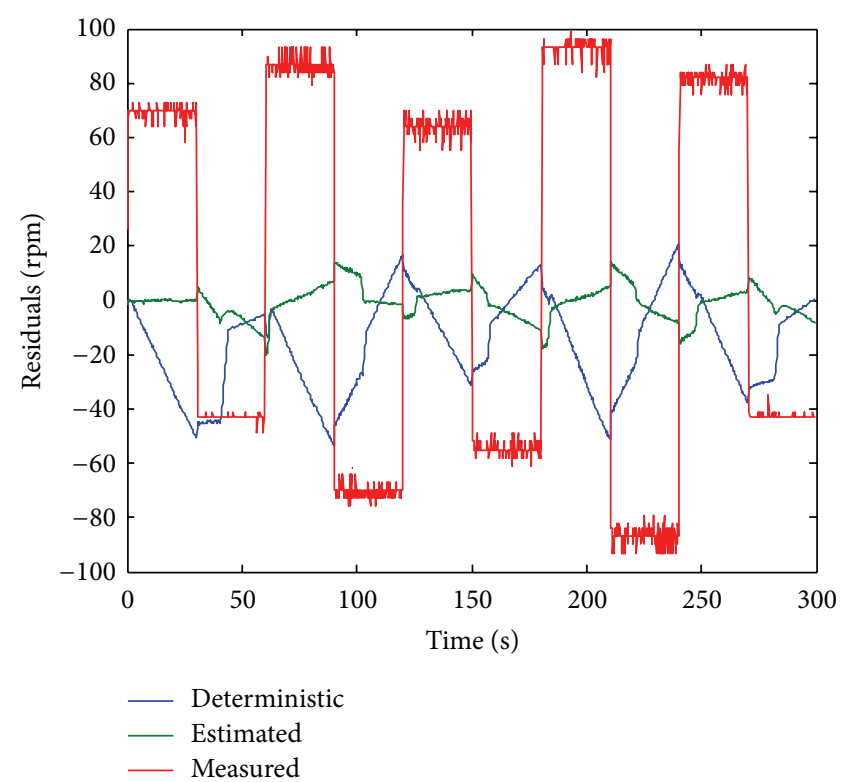

(b)

FIGURE 6: Estimation residuals of profiles 1 (a) and 2 (b).

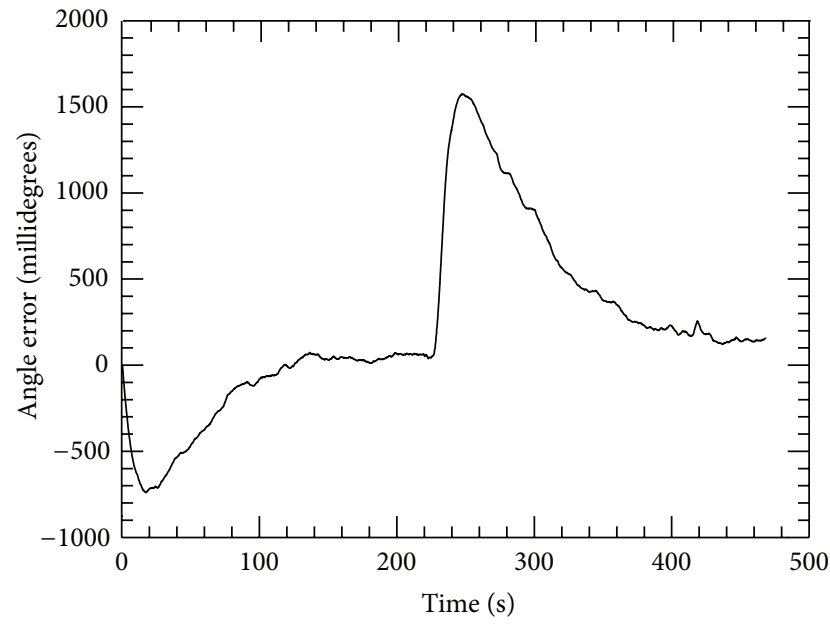

FIgURE 7: Attitude error during zero-speed crossing and with external disturbance (cooler fan).

where $\Omega$ is the table angular velocity, as measured by the FOG gyro, and $T_{w}$ is the wheel's reaction torque.

Figure 7 shows the attitude error with a null reference signal, while Figure 8 shows the commanded current, equal to the PID signal that is, $I=u$, where $u$ is the PID output. The maximum attitude error occurs during wheel's reversion, at elapsed time of 230 seconds, approximately. The torque generated by the fan could be estimated based on the angular momentum variation, resulting in $0.46 \times 10^{-3} \mathrm{Nm}$, and it is practically constant. The attitude error reaches 1.5 degrees after zero-speed crossing, followed by an error of 0.2 degree in steady state. From controller viewpoint, this means that the pointing requirement is no longer accomplished during

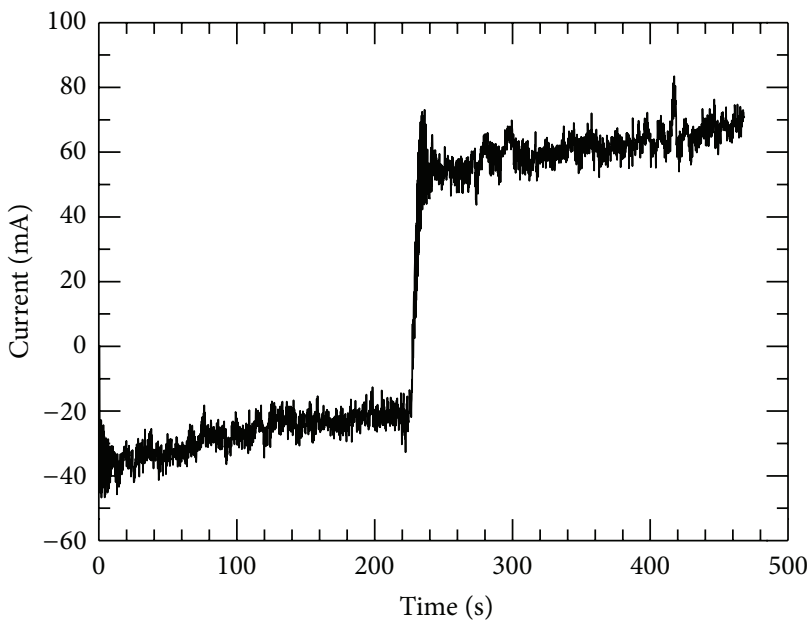

Figure 8: Control signal (motor current) under external disturbance.

zero-speed crossing. Most of the error is due to the long time the integral controller takes to compensate the fast changing in the friction torque during wheel reversion. As it will be shown, the DMC controller changes the control signal as quick as the friction torque, allowing the PID to respond only to the external disturbance torque.

4.2. Dynamic Model Compensator Control. Using a nonlinear controller to handle the zero-speed problem of the reaction wheel is a natural consequence of the fact that the mathematical model represents the behavior of the wheel reasonably well. It is, therefore, straightforward to use this model as a nonlinear compensator for the controller and to make the 


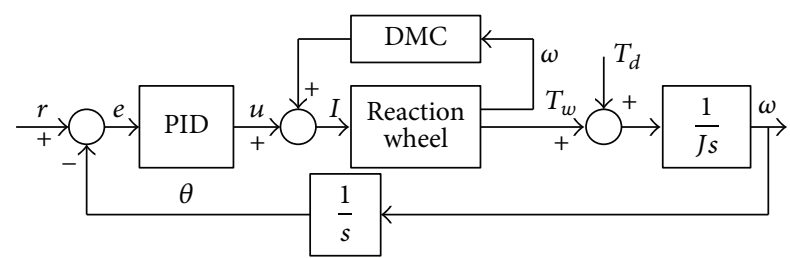

FIGURE 9: PID controller with RW dynamic model compensation.

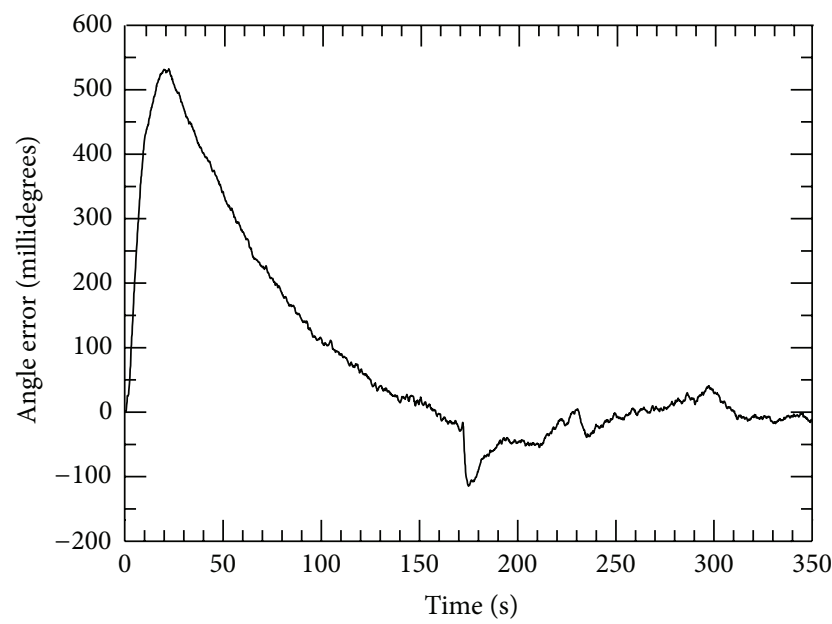

FIGURE 10: Attitude error of the air-bearing table with DMC control.

wheel action directly proportional to the PID signal [11]. Since the table responds to only an acceleration of the wheel, the control command will be in the form

$$
I=u+\frac{b}{k_{m}} \omega+\frac{\operatorname{sgn}(\omega)}{k_{m}}\left[c+d e^{-\omega^{2} / \omega_{s}^{2}}\right],
$$

where $u$ is the PID control signal. For a null wheel angular velocity the compensator takes the form

$$
I=u+\frac{(c+d) \operatorname{sgn}(u)}{k_{m}} .
$$

Figure 9 shows a simplified block diagram of the Dynamic Model Compensator (DMC) control. The new controller was tested under the same condition as the torque mode control but incorporating the dynamic compensation. As can be seen in Figures 10 and 11 (analogous to Figures 7 and 8 ) the error was almost negligible, with a maximum deviation of only 0.1 degree during wheel reversion, and it took about $20 \mathrm{~s}$ to reach the steady state. The large error of almost 0.6 degree is due to the initial step response of the control at the beginning of the experiment and will not be considered as a steady error. The control signal is shown in black in Figure 11 and separated in its two components: the friction dynamic compensator (in red) and the PID signal (blue curve). It is clear in this graph that the PID control is approximately constant, as it would be expected due to the disturbing torque of the fan. The PID controller gains were kept identical to those used previously, although they could

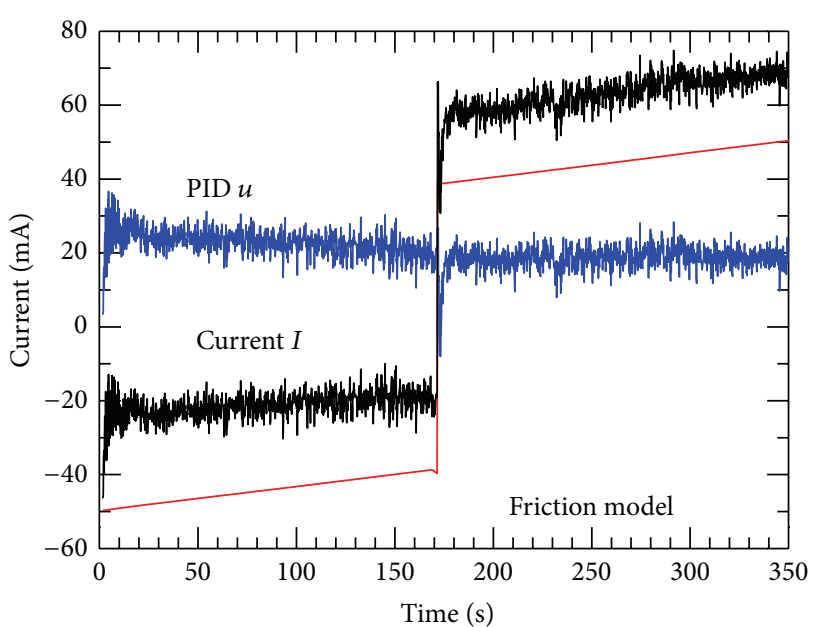

FIgURE 11: PID controller with RW dynamic model compensation.

be adjusted in order to achieve a better performance, since the dynamics is now almost linear due to the model compensator.

The effect of the Stribeck friction is barely seen in Figure 11, which indicates that this friction is not so significant for the wheel's behavior. In fact, the same experiment was carried out without the Stribeck friction model in the DMC (not shown in this paper), which showed similar results. However, it is not recommended to simply neglect the Stribeck factor, since it introduces some sort of hysteresis that should be important during motor starting and reversing.

\section{Conclusions}

This paper presented a computational and mathematical model for an off-the-shelf reaction wheel [21], obtained from nonlinear models of Coulomb, viscous, and Stribeck frictions, based on testing and experimental measurements of the behavior of the wheel. Previous work did not include Stribeck friction, and the values of the parameters of friction (Coulomb and viscous) were obtained deterministically [2, 3]. Estimation of Stribeck friction via extended Kalman filtering was attempted [5] but resulted nonconclusive due to the limited data used. Another work used the LuGre Model [13] with extended Kalman filter but only at simulation level (no actual data).

Based on the more complete proposed model, a nonlinear estimation of states and parameters by the method of least squares, using data from two experiments: one with sinusoidal profile and another with positive and negative levels, where the transitions by zero were exercised numerous times (24 times in the sinusoidal profile 1 and 9 times in the stepwise profile 2) were accomplished. As expected, degraded performance of the models in the crossings by zero was noted, but with better fit of statistical method. A nonlinear Dynamic Model Compensator (DMC) for the reaction wheel control was then implemented in order to make the wheel behavior linear. The controller showed improved performance in this new condition and reached the maximum error of only 0.1 degrees at zero-speed crossing. 
The DMC presented also smooth responses near zero, as expected, with errors smaller than the ones presented with the deterministic parameter estimation method [4]. Due to this, the compensator significantly reduced the nonlinear effects that occur in the response of the wheel during the reversals of direction, avoiding the model discretization and decreasing the complexity of the control synthesis in this type of actuator. Future works suggest the use of this model in a control system of position (angle) or angular velocity and corresponding performance comparisons in terms of response time, performance, and accuracy.

\section{Disclosure}

The authors are solely responsible for the printed material included in this paper.

\section{Acknowledgment}

The authors thank the project FUNDEP-FINEP-SIA-11382 $* 3$ which provided the support for acquiring equipment used in the experiments.

\section{References}

[1] B. Robertson and E. Stoneking, "Satellite GN\&C Anomaly Trends," in Proceedings of the Annual AAS Rocky Mountain Guidance and Control Conference, vol. 113 of Advances in the Astronautical Sciences, pp. 531-542, 2003.

[2] V. Carrara and P. G. Milani, "Control of one-axis air bearing table equipped with gyro and reaction wheel," in Proceedings of the 5th Brazilian Symposium on Inertial Engineering (SBEIN '07), Rio de Janeiro, Brazil, November 2007.

[3] V. Carrara, "Comparison between means of attitude control with reaction wheels," in Proceedings of the 6th Brazilian Symposium on Inertial Engineering (SBEIN '10), Rio de Janeiro, Brazil, November 2010.

[4] V. Carrara, R. Siqueira, and D. Oliveira, "Speed and current mode strategy comparison in satellite attitude control with reaction wheels", in Proceedings of the 21st International Congress of Mechanical Engineering (COBEM '11), Natal, Brazil, October 2011.

[5] D. C. Fernandes, H. K. Kuga, V. Carrara, and R. A. Romano, "A model of a reaction wheel with friction parameters estimation by Kalman filter," in Proceedings of the 7th Brazilian Congress of Mechanical Engineering (CONEM '12), Sao Luis, Brazil, August 2012.

[6] M. L. B. Moreira, R. V. F. Lopes, and H. K. Kuga, "Estimation of torque in a reaction wheel using a Bristle model for friction," in Proceedings of the 18th International Congress of Mechanical Engineering, Ouro Preto, Brazil, November 2005.

[7] G. Shengmin and H. Cheng, "A comparative design of satellite attitude control system with reaction wheel," in Proceedings of the 1st NASA/ESA Conference on Adaptive Hardware and Systems (AHS '06), pp. 359-362, June 2006.

[8] H. Olsson, K. J. Åström, C. Canudas de Wit, M. Gafvert, and P. Lischinsky, "Friction models and friction compensation," European Journal of Control, vol. 4, no. 3, pp. 176-195, 1998.

[9] C. Canudas de Wit and S. S. Ge, "Adaptive friction compensation for systems with generalized velocity/position friction dependency," in Proceedings of the 36th IEEE Conference on Decision and Control, pp. 2465-2470, San Diego, Calif, USA, December 1997.

[10] C. Canudas de Wit, H. Olsson, K. J. Astrom, and P. Lischinsky, "New model for control of systems with friction," IEEE Transactions on Automatic Control, vol. 40, no. 3, pp. 419-425, 1995.

[11] C. Canudas de Wit and P. Lischinsky, "Adaptive friction compensation with partially known dynamic friction model," International Journal of Adaptive Control and Signal Processing, vol. 11, no. 1, pp. 65-80, 1997.

[12] K. J. Åström and C. Canudas-de-Wit, "Revisiting the LuGre friction model," IEEE Control Systems Magazine, vol. 28, no. 6, pp. 101-114, 2008.

[13] V. Carrara, A. G. Silva, and H. K. Kuga, "A dynamic friction model for reaction wheels," in Proceedings of the 1st IAA Conference on Dynamics and Control of Space Systems (DyCoSS '12), vol. 145 of Advances in the Astronautical Sciences, pp. 352343, Porto, Portugal, March 2012.

[14] D. Karnopp, "Computer simulation of slip-stick friction in mechanical dynamic systems," Transactions of the ASME, Journal of Dynamic Systems, Measurement and Control, vol. 107, no. 1, pp. 100-103, 1985.

[15] F. Al-Bender, V. Lampaert, and J. Swevers, "The generalized Maxwell-slip model: a novel model for friction simulation and compensation," IEEE Transactions on Automatic Control, vol. 50, no. 11, pp. 1883-1887, 2005.

[16] R. M. Hirschorn and G. Miller, "Control of nonlinear systems with friction," IEEE Transactions on Control Systems Technology, vol. 7, no. 5, pp. 588-595, 1999.

[17] J. Amin, B. Friedland, and A. Harnoy, "Implementation of a friction estimation and compensation technique," IEEE Control Systems Magazine, vol. 17, no. 4, pp. 71-76, 1997.

[18] R. H. A. Hensen, M. J. G. van de Molengraft, and M. Steinbuch, "Frequency domain identification of dynamic friction model parameters," IEEE Transactions on Control Systems Technology, vol. 10, no. 2, pp. 191-196, 2002.

[19] A. Ramasubramanian and L. R. Ray, "Comparison of EKBFbased and classical friction compensation," Transactions of the ASME, Journal of Dynamic Systems, Measurement and Control, vol. 129, no. 2, pp. 236-242, 2007.

[20] B. Armstrong-Hélouvry, P. Dupont, and C. C. de Wit, "A survey of models, analysis tools and compensation methods for the control of machines with friction," Automatica, vol. 30, no. 7, pp. 1083-1138, 1994.

[21] J. A. A. Engelbrecht, User's Manual for the SunSpace Reaction Wheel and Gyroscope Subsystem, SunSpace, Matieland, South Africa, 2005, (SS01-106000).

[22] C. L. Lawson and R. J. Hanson, Solving Least Squares Problems, Prentice-Hall, Englewood Cliffs, NJ, USA, 1974, Prentice-Hall Series in Automatic Computation.

[23] H. K. Kuga, Orbit determination of artificial satellites by estimation techniques and state smoothing techniques [Ph.D. dissertation], INPE, Sao Jose dos Campos, Brazil, 1989, (INPE4959-TDL/388).

[24] C. Canudas de Wit and K. J. Åström, "Adaptive friction compensation in DC-motor drivers," IEEE Journal of Robotics and Automation, vol. 3, no. 6, pp. 681-685, 1987. 


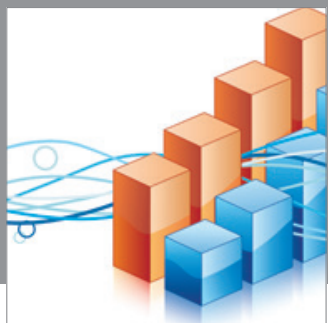

Advances in

Operations Research

mansans

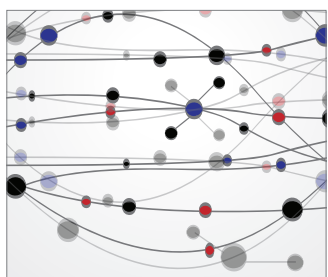

The Scientific World Journal
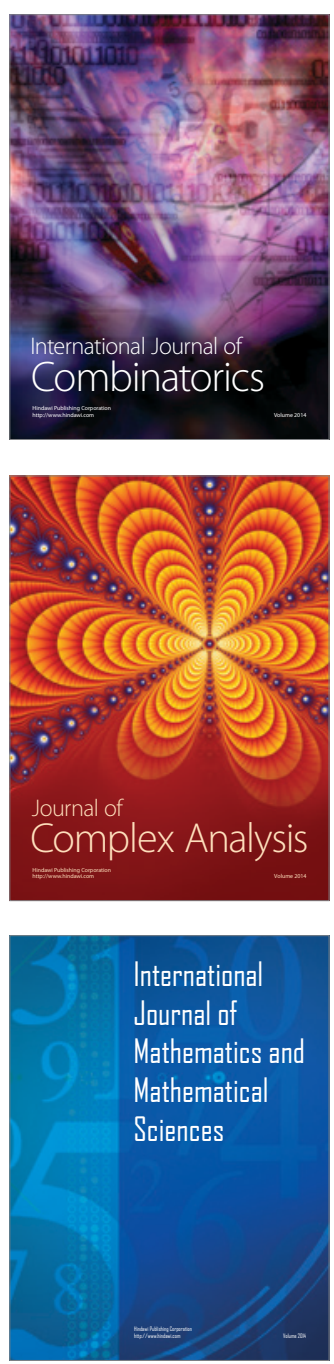
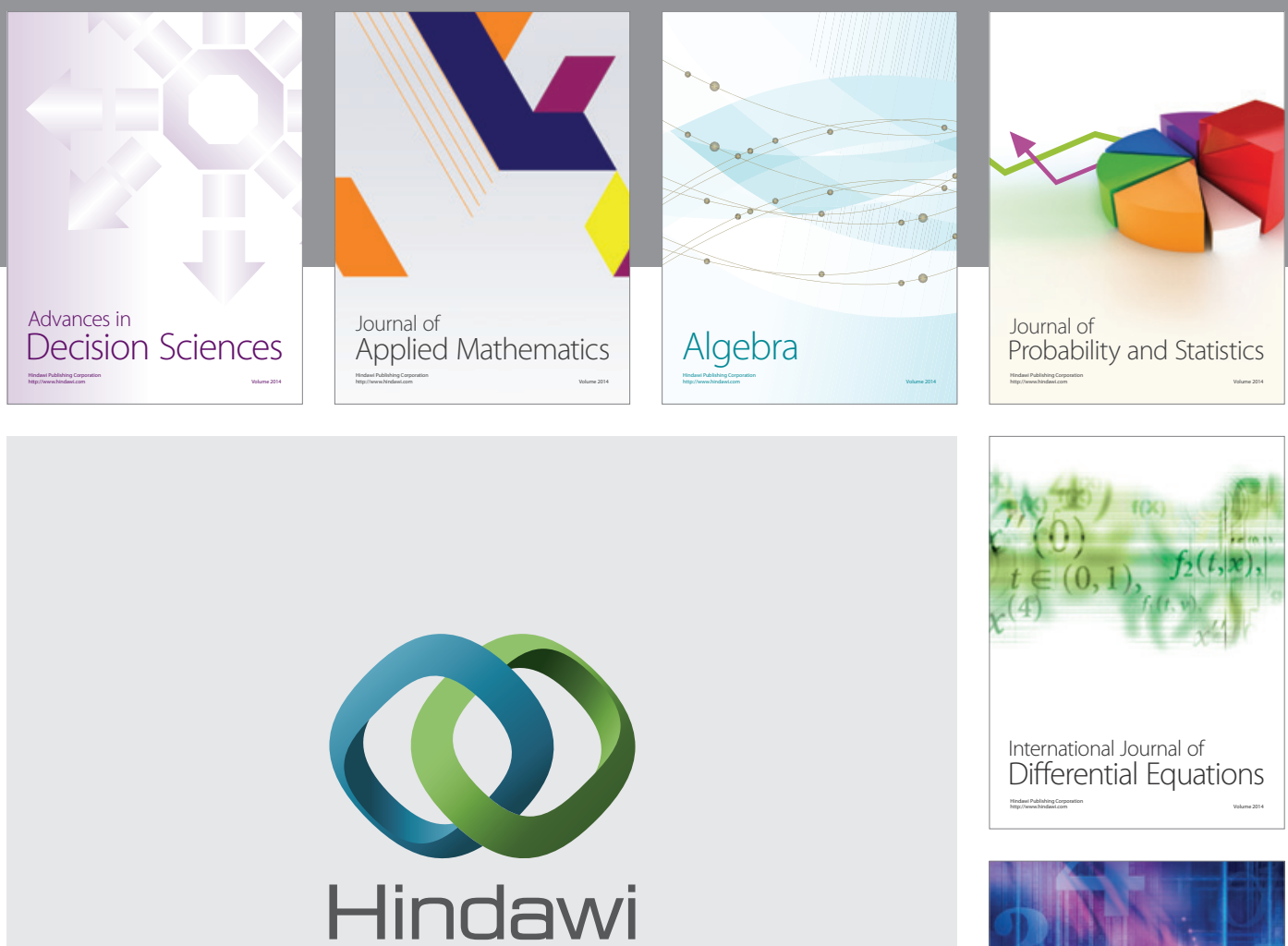

Submit your manuscripts at http://www.hindawi.com
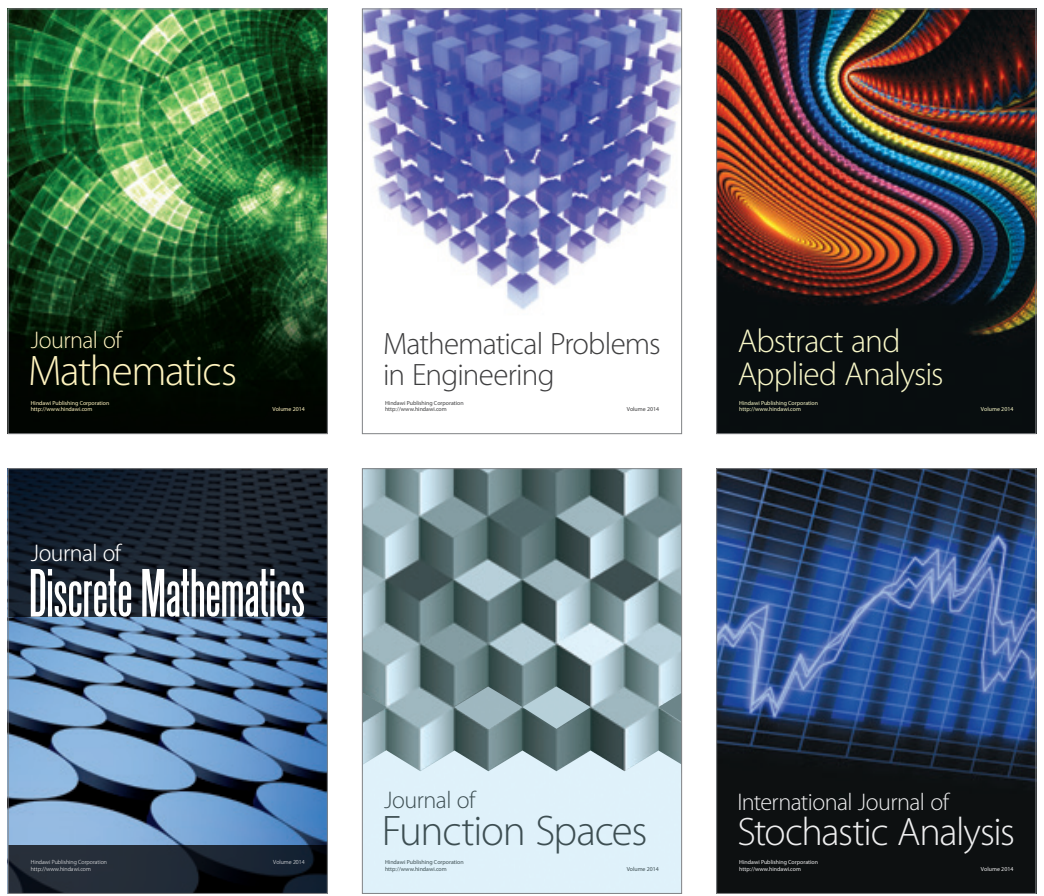

Journal of

Function Spaces

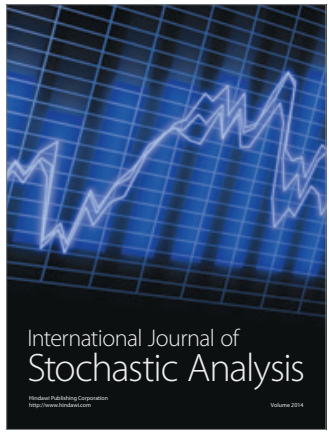

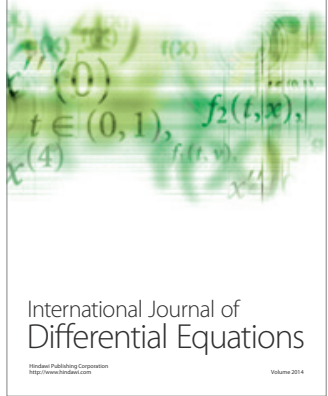
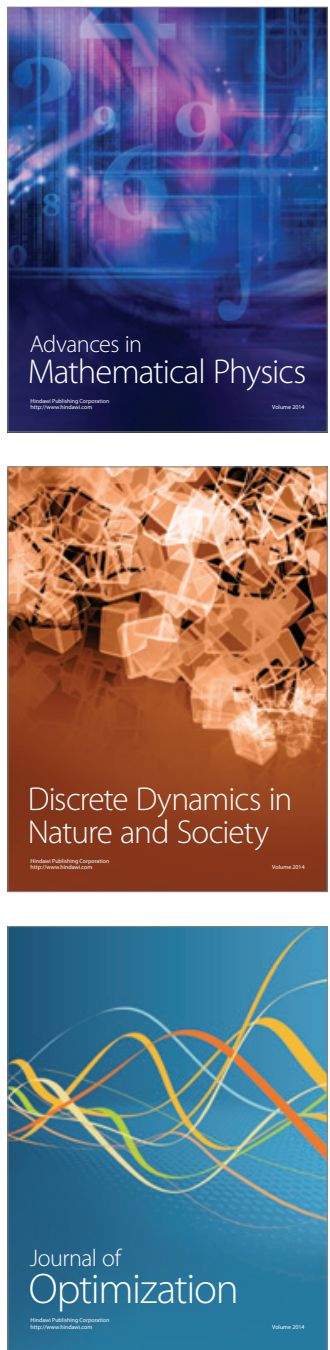